\title{
Notch signaling in the collecting duct regulates renal tubulointerstitial fibrosis induced by unilat- eral ureteral obstruction in mice
}

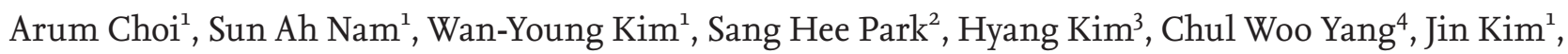 \\ and Yong Kyun $\mathrm{Kim}^{1,4}$
}

\author{
${ }^{1}$ Department of Anatomy and Cell \\ Death Disease Research Center, \\ College of Medicine, The Catholic \\ University of Korea, Seoul; ${ }^{2}$ Institute \\ of Clinical Medicine Research \\ of Bucheon St. Mary's Hospital, \\ Bucheon; ${ }^{3}$ Division of Nephrology, \\ Department of Medicine, Kangbuk \\ Samsung Hospital, Sungkyunkwan \\ University School of Medicine, Seoul; \\ ${ }^{4}$ Department of Internal Medicine, \\ College of Medicine, The Catholic \\ University of Korea, Seoul, Korea \\ Received: July 15, 2016 \\ Revised : September 22, 2016 \\ Accepted: September 24, 2016

\section{Correspondence to} \\ Jin Kim, M.D. \\ Department of Anatomy and \\ Cell Death Disease Research \\ Center, College of Medicine, The \\ Catholic University of Korea, 222 \\ Banpo-daero, Seocho-gu, Seoul \\ 06591, Korea \\ Tel: +82-2-2258-7258 \\ Fax: +82-2-2536-3110 \\ E-mail:jinkim@catholic.ac.kr
}

Background/Aims: Mind bomb-1 (Mib1) encodes an E3 ubiquitin ligase, which is required for the initiation of Notch signaling. Recently, it was demonstrated that the renal collecting duct plays an important role in renal fibrosis. Here, we investigated the role of Notch signaling in renal fibrosis using conditional knockout mice with the specific ablation of Mibı in renal collecting duct principal cells. Methods: Mib1-floxed mice (Mibf ${ }^{f / 5}$ ) were crossed with aquaporin 2 (AQP2)-Cre mice in order to generate principal cell-specific Mibı knockout mice (Mibf ${ }^{f / f}$ :AQP2-Cre ${ }^{+}$). Unilateral ureteral obstruction (UUO) was performed, and mice were sacrificed 7 days after UUO.

Results: After performing the UUO, renal tubulointerstitial fibrosis and the expression of transforming growth factor $\beta$ were markedly enhanced in the obstructed kidneys of Mibf/s mice compared with the sham-operated kidney of $\mathrm{Mibf} / \mathrm{f}$ mice. These changes were shown to be even more pronounced in the obstructed kidneys of Mibf f/f:AQP2-Cre ${ }^{+}$mice than in those of the Mibf f/f mice . Furthermore, the number of TUNNEL-positive cells in renal collecting duct was higher in the obstructed kidneys of $\mathrm{Mibs}^{\mathrm{f} / \mathrm{f}}: \mathrm{AQP}_{2}-\mathrm{Cre}^{+}$mice than in the kidneys of Mibf $f / f$ mice.

Conclusions: Notch signaling in the renal collecting duct plays an important role in the regulation of renal tubulointerstitial fibrosis and apoptosis after UUO.

Keywords: Mibı; Notch; Renal fibrosis; Kidney collecting duct; Ureteral obstruction

\section{INTRODUCTION}

Progressive renal tubulointerstitial fibrosis occurs in virtually every type of chronic kidney disease, and it is characterized by excessive accumulation of fibroblasts/ myofibroblasts with the increased production and deposition of extracellular matrix (ECM), including collagen, fibronectin, and other related fibrogenic molecules [13]. Although the activation of fibroblasts/myofibroblasts is generally considered the main cause of the excessive production and deposition of ECM in the pathogenesis of renal tubulointerstitial fibrosis, the underlying mechanisms remain unclear $[4,5]$.

The Notch signaling pathway is an evolutionary conserved cell-cell communication mechanism. Four receptors, Notch1-4, and five ligands, delta-like1, 3, 4 (Dllı, 3, 4), and Jaggedı and 2 (Jag1, 2), involved in this signaling pathway can be found in mammals [6]. The endocytosis 
of Notch ligands in the signal sending cells is necessary for the initiation of notch signaling. In mammals, four E3 ubiquitin ligases, neuralized-1 (Neur1) and Neur2, and mind bomb-1 (Mib1) and Mib2, are known to regulate the endocytosis of Notch ligands [7]. Among these E3 ubiquitin ligases, only Mibı has an obligatory role in the activation of Jag- as well as DII-mediated Notch signaling in mammalian development, while Neurı, Neur2, and Mib2 activity is dispensable $[8,9]$. Thus, genetic mutation of Mibı represents an excellent model for the elucidation of the role of Notch signaling [10,11]. In kidney diseases models, Notch signaling pathway is reported to be involved in renal fibrosis-related diseases, such as diabetic nephropathy and focal segmental glomerulosclerosis [12]. Notch signaling pathway plays an important role in the development of tubulointerstitial fibrosis as well [13]. Several studies showed that the principal cells of renal collecting duct may play a role in the development of tubulointerstitial fibrosis [14-17]. However, their role remains unclear [17]. Unilateral ureteral obstruction (UUO) is the most widely used animal model of chronic kidney disease, with renal tubulointerstitial fibrosis $[18,19]$.

In this study, we determined the role of Notch signaling in principal cells of renal collecting duct in renal tubulointerstitial fibrosis induced by UUO. Principal cell-specific Mibı knockout mice that underwent UUO surgery were used in our experiments.

\section{METHODS}

\section{Animals}

To generate mice with a Mibı deletion specifically in principal cell of collecting duct (Mibı-floxed mice [Mib$\left.{ }^{\mathrm{f} / f}\right]$ :aquaporin $\left.2[\mathrm{AQP} 2]-\mathrm{Cre}^{+}\right)$, we crossed Mibf f/f mice (kindly provided by Prof. Kong, Seoul National University, Seoul, Republic of Korea) with AQP2-cre mice (Stock No. 006881, purchased from The Jackson Laboratory, West Grove, PA, USA). All mice were crossed on a C57BL6 background and only male mice were used in the study. UUO was performed as described previously [18]. Briefly, mice were anesthetized with zoletil and the left ureter was exposed via a left dorsal incision. The mid-ureter was then obstructed using a two-point ligation with silk sutures. The sham-operated mice underwent the same procedure with the exception of obstruction of the left ureter and used as controls. Mice were sacrificed at 7 days after UUO. After anesthetized, the animals were perfused with phosphate buffered saline (PBS; pH 7.4), and then fixed with $2 \%$ paraformaldehyde-lysine-periodate solution, which was administered through the heart for 10 minutes. After perfusion, the kidneys were removed and cut into 1 to $2 \mathrm{~mm}$ thick slices, which were further fixed by immersion in the same fixative overnight at $4^{\circ} \mathrm{C}$. All the experimental procedures were performed according to the Animal Care and Ethics Legislation and the study was approved by the Animal Care Committee of Bucheon St. Mary's Hospital.

\section{Antibodies}

The antibodies used in this study were as follows: AQP2 (Millipore, Billerica, MA, USA), Notchı (Abcam, Cambridge, UK), fibronectin (DAKO, Glostrupp, Denmark), collagen IV (SouthernBiotech, Birmingham, AL, USA), fibroblast-specific protein 1 (FSP1, Thermo Scientific, Cheshire, UK), transforming growth factor $\beta$ (TGF- $\beta$, R\&D systems, Minneapolis, MN, USA), Smad4 (Santa Cruz Biotechnology, Santa Cruz, CA, USA), c-Myc (Santa Cruz Biotechnology), and glyceraldehyde 3-phosphate dehydrogenase (Santa Cruz Biotechnology) were used. Apoptosis was detected using an ApopTag Peroxidase in situ Apoptosis Detection Kit (Millipore).

\section{Immunohistochemical analysis}

For single post-embedding immunohistochemical staining (IHC), after fixation, kidney was embedded in wax and cut transversely at a thickness of $4 \mu \mathrm{m}$ using a microtome. Some kidney sections were processed and stained with periodic acid-Schiff (PAS) stain or Masson's trichrome stain. Other sections were processed for post-embedding immunohistochemistry analysis. These tissue sections were hydrated with graded ethanol and rinsed in tap water. After dewaxing, the sections were incubated with retrieval solution for 10 minutes by microwave and then washed in tap water. They were incubated with methanolic $\mathrm{H}_{2} \mathrm{O}_{2}$ for 30 minutes for endogenous peroxidase blocking. After the process, they were incubated with $0.5 \%$ Triton X-100/PBS solution for 15 minutes and they were rinsed with PBS. The nonspecific binding sites were blocked with normal donkey serum diluted 1:10 in PBS for 1 hour and subsequently incubated overnight 
with a primary antibody at $4{ }^{\circ} \mathrm{C}$. Next day, after rinsing in PBS, the sections were incubated for 2 hours in peroxidase-conjugated donkey anti-mouse or anti-rabbit immunoglobulin G (IgG; Jackson Immuno Research Lab., West Grove, PA, USA) and washed again with 0.05 $\mathrm{M}$ Tris buffer ( $\mathrm{pH}$ 7.6). For detection, the sections were treated with $0.05 \% 3,3$ '-diaminobenzidine (DAB) and $0.01 \% \mathrm{H}_{2} \mathrm{O}_{2}$ mixture. The sections were washed with distilled water, dehydrated with graded ethanol and xylene, mounted in Canada balsam, and examined by light microscopy.

For multiple post-embedding IHC, after DAB colorizing, tissue sections were treated with methanolic $\mathrm{H}_{2} \mathrm{O}_{2}$ for 30 minutes to remove any peroxidase remaining from the first staining. The sections were incubated with the other primary antibody. After a wash in PBS, the sections were incubated for 2 hours with peroxidase-conjugated donkey anti-rabbit IgG (Jackson Immuno Research Lab.). For detection of peroxidase, Vector SG (Vector Laboratories, Burlingame, CA, USA) was used as a chromogen to produce a grayish blue color, which is easily distinguished from the brown staining produced by DAB. The sections were washed with distilled water, dehydrated with graded ethanol and xylene, mounted in Canada balsam, and examined by light microscopy.

\section{Western blot analysis}

The kidney was homogenized in boiling lysis buffer $(1 \%$ sodium dodecyl sulfate [SDS], $1 \mathrm{mM}$ sodium orthovanadate, and $10 \mathrm{mM}$ Tris, $\mathrm{pH}$ 7.4) and the protein concentration was determined with the BCA Protein assay kit (Pierce Biotechnology Inc., Rockford, IL, USA). Equal amounts of the protein were separated on SDS-polyacrylamide gel. The gel was transferred onto a nitrocellulose membrane. For immunodetection, the nonspecific binding sites were blocked with PBS that containing $0.1 \%$ Tween-20 and 5\% skim milk and then the blots were incubated overnight in the same solution with the primary antibody. The blots were washed and then incubated with a secondary antibody conjugated to horseradish peroxidase (Jackson Immuno Research Lab.) and the blots were visualized using a Western blotting luminol reagent kit (Santa Cruz Biotechnology).

\section{Cell counting and statistics}

The deposition of immunohistochemistry results and cell counting were measured by JNOPTIC Image Analysis Software OpTIC Eye (Seoul, Korea). The values are expressed as percentage of the total number of cells in the respective segments. Values are presented as the mean \pm SE. Data were compared between groups using an unpaired $t$ test and Microsoft Excel 2007 (Microsoft, Redmond, WA, USA). $p$ values less than 0.05 were considered significant.

\section{RESULTS}

\section{The expression of Notch1}

The functional role of Notch signaling in the principal cells of renal collecting duct in renal tubulointerstitial fibrosis, was investigated by generating principal cell-specific conditional Mibr knockout mice (Mibrtf :AQP2-Cre ${ }^{+}$.

First, we investigated Notch 1 expression in sham-operated and UUO groups. The number of Notch 1-positive cells in Miby flf:AQP2-Cre ${ }^{+}$mice sham-operated mice was lower than that in Mibff/s mice. Its expression was higher in tubular epithelial cells after UUO. The number of Notch 1-positive cells in Mibff/s:AQP2-Cre ${ }^{+}$mice was lower than in Mibff/s mice after UUO (Fig. 1A). Following this, we performed double IHC staining using antibodies for AQP2 (marker of the principal cells of collecting duct) and Notch 1 . In both sham-operated control mouse kidneys and the obstructed kidneys, Notch 1 expression in AQP2-positive principal cells was considerably lower in Mibff/f:AQP2-Cre ${ }^{+}$mice than in Miby f/f mice (Fig. $1 \mathrm{~B}$ ), suggesting that the Notch signaling pathway is selectively inhibited in the principal cells of Mib${ }_{1}^{f} / f_{:} \mathrm{AQP} 2-\mathrm{Cre}^{+}$mouse collecting ducts.

\section{Principal cell-specific Mibr deletion enhances tubu- lointerstitial fibrosis after UUO}

The effects of principal cells-specific deletion of Mib1 in renal tubulointerstitial fibrosis induced by UUO were investigated by PAS and Masson's trichrome staining. ECM deposition within the tubulointerstitium at day 7 after UUO was higher in Mibfl/s mice than in sham-operated Mibff/s mice, and the deposition was even greater in the obstructed kidneys of Mibf/f:AQP2-Cre ${ }^{+}$mice (Fig. 2). Similar data were obtained by analyzing fibronectin expression using Western blot (Fig. 3A). The expression 

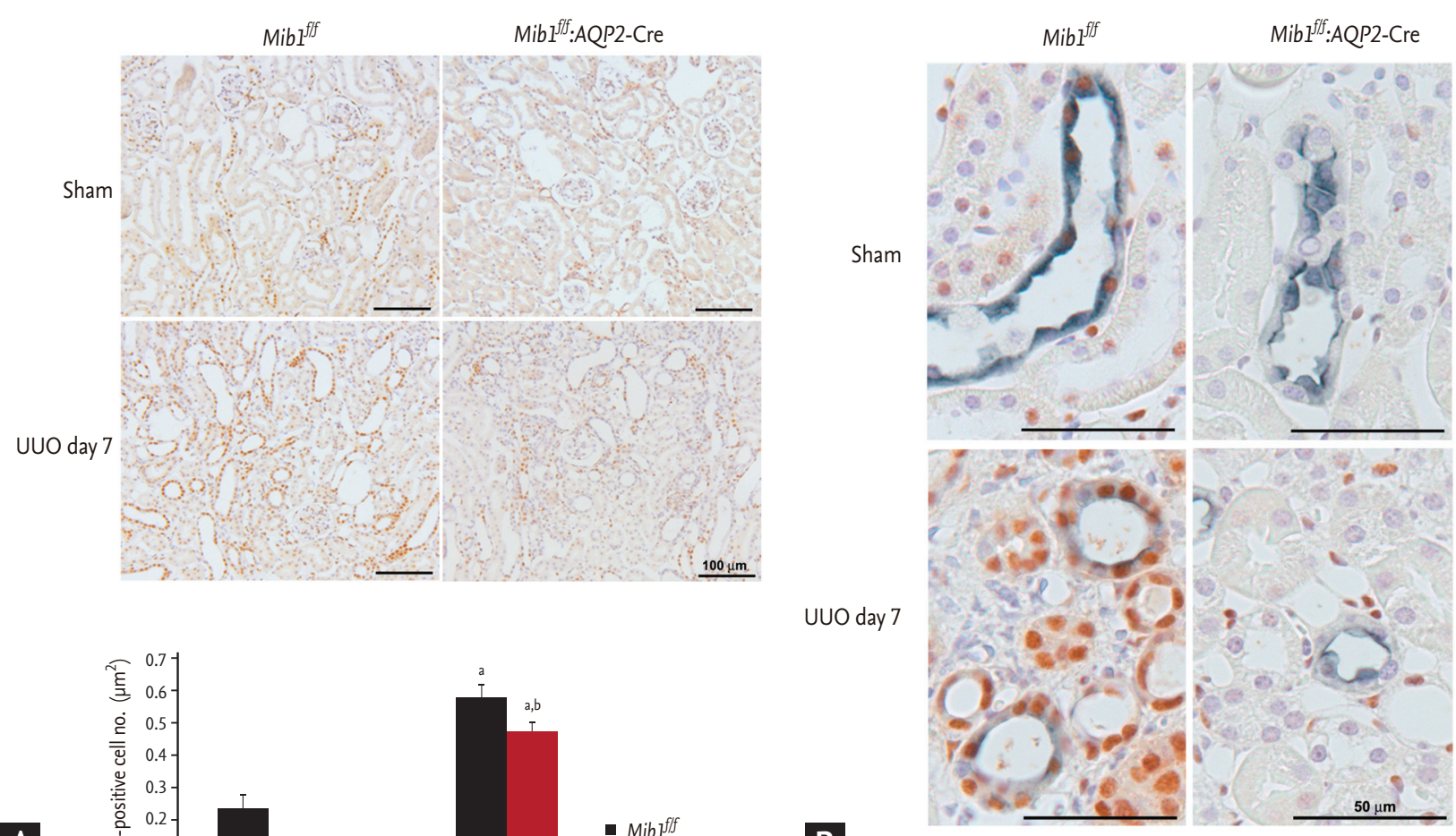

A

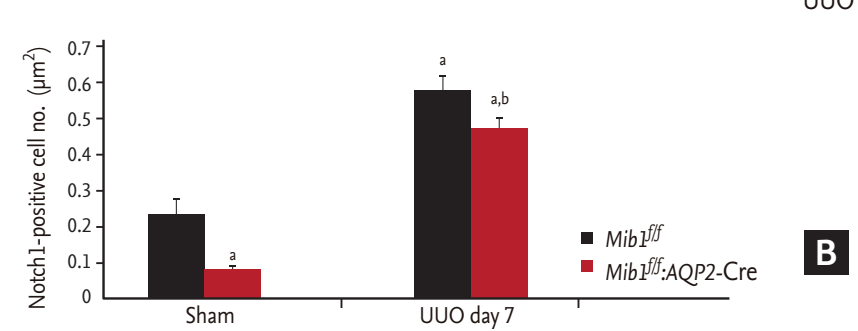

.

Figure 1. (A) Notch 1 expression and the quantification of Notch 1-positive cells. Scale bars: $100 \mu \mathrm{m}$. The results are presented as mean \pm standard error. (B) Aquaporin 2 (AQP2, blue) and Notch 1 (brown) sample staining. Scale bars: $50 \mu \mathrm{m}$. Mibr $\mathrm{f} / \mathrm{f}$, mind bomb-1 (Mibi)-floxed mice; UUO, unilateral ureteral obstruction. ${ }^{\mathrm{a}} \mathrm{p}<0.05 \mathrm{vs}$. MibI ${ }^{\mathrm{f} / \mathrm{s}}$ Sham, ${ }^{\mathrm{b}} \mathrm{p}<0.05 \mathrm{vs}$. Mibi $/ \mathrm{f}^{\mathrm{U}} \mathrm{UUO}$.

of type IV collagen was higher in the obstructed kidneys of Mibyff:AQP2-Cre ${ }^{+}$mice than in those of Miby ffs mice (Fig. 3B). Furthermore, we performed the IHC staining for $\mathrm{FSP} 1$, as a marker of fibroblast. FSP1 expression was significantly increased in the obstructed kidneys of Mibrff/:AQP2-Cre ${ }^{+}$mice compared with that in the Mibrfff mice (Fig. $3 \mathrm{C}$ ).

\section{Principal cell-specific Mibr deletion induces the expression of TGF- $\beta 1$ and Smad 4}

TGF- $\beta$ and Smad signaling pathways are important mechanisms in renal fibrosis. IHC analyses showed that TGF- $\beta 1$ expression was upregulated in the obstructed kidneys of Mibff/s:AQP2-Cre ${ }^{+}$mice after UUO (Fig. 4A). Western blot analyses revealed that TGF- $\beta 1$ and Smad 4 protein expression levels were considerably higher in the obstructed kidneys of Mibff/s:AQP2-Cre+ mice than in the kidneys of Mibff/s mice (Fig. ${ }_{4} \mathrm{~B}$ and $4 \mathrm{C}$ ).

\section{Principal cell-specific Mibı deletion enhances apop- tosis after UUO}

The role of Mibr in cellular apoptosis after UUO was investigated using the TUNNEL (Terminal deoxynucleotide transferase dUTP Nick End Labeling) assay. While a very low number of TUNNEL-positive cells was detected in the sham-operated control mice, this number increased 7 days after UUO and was observed to be even higher in the obstructed kidneys of Mibfff:AQP2-Cre ${ }^{+}$ mice than in the obstructed kidneys of Mibff/s mice (Fig. 5A).

Additionally, we showed that the number of TUNNELpositive cells in AQP2-positive collecting ducts increased more in Miby f/f:AQP2-Cre ${ }^{+}$mice than in Miby f/s mice, and it was even higher in Mibfff:AQP2-Cre ${ }^{+}$mice after UUO. However, the number of TUNNEL-positive cells in the non-collecting duct decreased after UUO in Mibfff:AQP2$\mathrm{Cre}^{+}$mice (Fig. 5B). The expression of c-Myc was analyzed, and it was a shown to be significantly higher in the obstructed kidneys of Mibfff:AQP2-Cre ${ }^{+}$mice than 

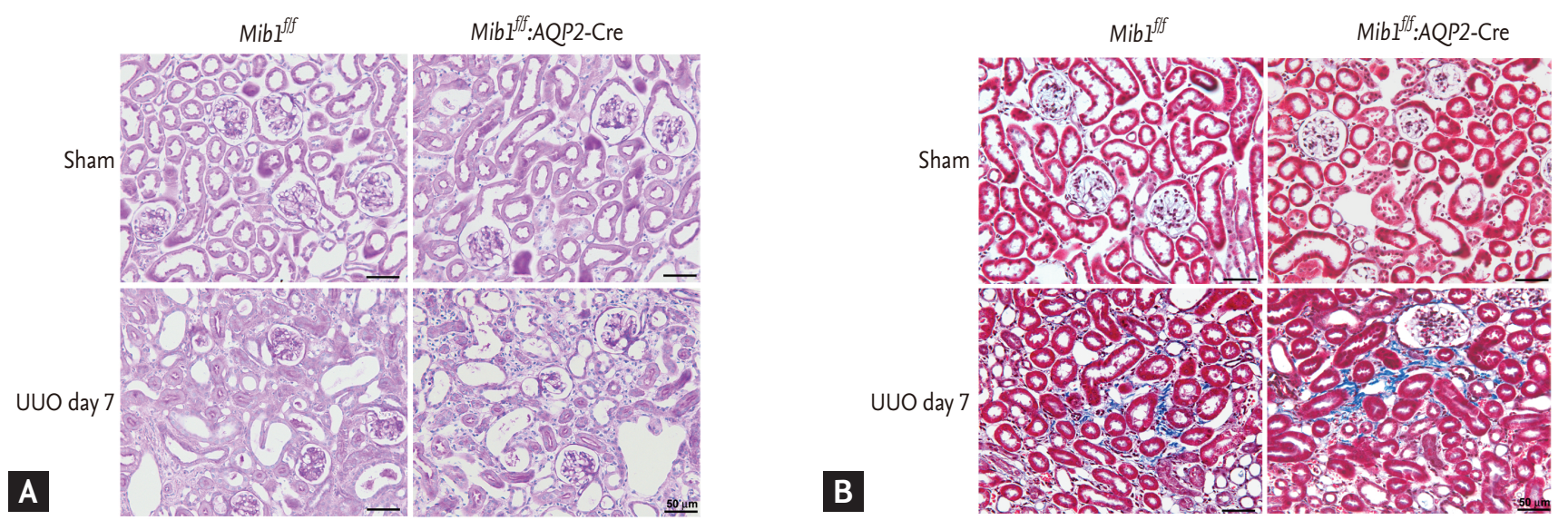

Figure 2. (A) Periodic acid-Schiff and (B) Masson's trichrome staining of the investigated samples. Scale bars: $50 \mu \mathrm{m}$. Mibr f/f, mind bomb-1 (Mib1)-floxed mice; AQP2, aquaporin 2; UUO, unilateral ureteral obstruction.

\begin{tabular}{|c|c|c|c|}
\hline \multicolumn{2}{|c|}{ Sham } & \multicolumn{2}{|c|}{ UUO day 7} \\
\hline Mibiff/ & $\begin{array}{c}\text { Mibffff: } \\
\text { AQP2-Cre }\end{array}$ & Mibifff & $\begin{array}{c}\text { Mib1 flf: } \\
\text { AQP2-Cre }\end{array}$ \\
\hline
\end{tabular}
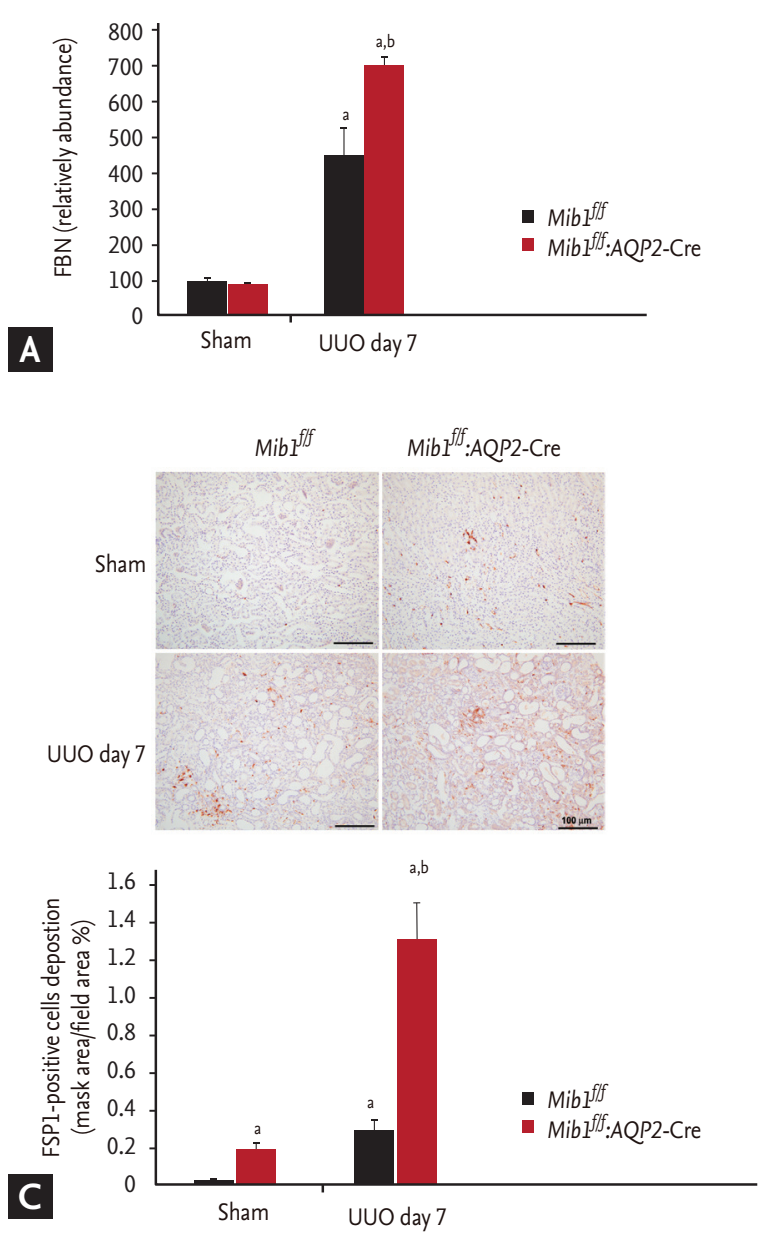

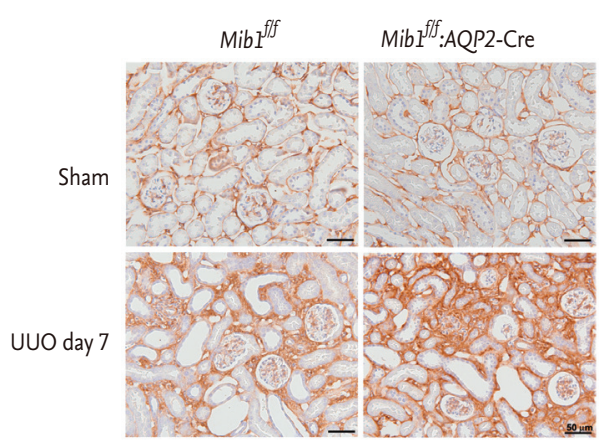

B

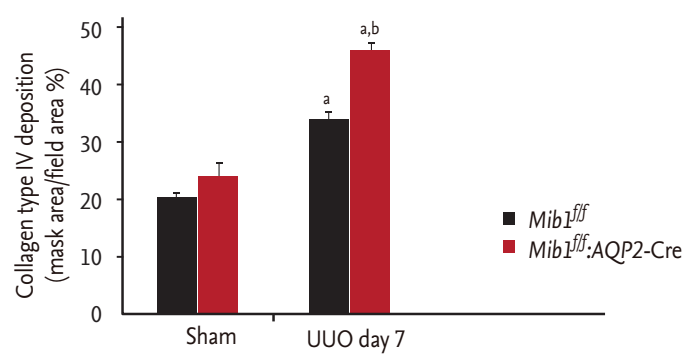

Figure 3. (A) Representative fibronectin (FBN) immunoblot results. (B) Collagen IV immunostaining and the quantification of collagen IV deposition (mask area/field area \%). Scale bars: $50 \mu \mathrm{m}$. (C) Fibroblast-specific protein 1 (FSP1) immunostaining and the quantification of FSP1 deposition (mask area/field area \%). Scale bars: $100 \mu \mathrm{m}$. The results are represented as mean \pm standard error. Mibf $f / f$, mind bomb1 (Mib1)-floxed mice; $A Q P_{2}$, aquaporin 2; UUO, unilateral ureteral obstruction; GAPDH, glyceraldehyde 3-phosphate dehydrogenase. ${ }^{\mathrm{a}} \mathrm{p}<0.05 \mathrm{vs}$. Mibs ${ }^{\mathrm{f}}$ Sham, ${ }^{\mathrm{b}} \mathrm{p}<0.05 \mathrm{vs} . \mathrm{Mibs}{ }^{\mathrm{f} / \mathrm{f}}$ UUO. 

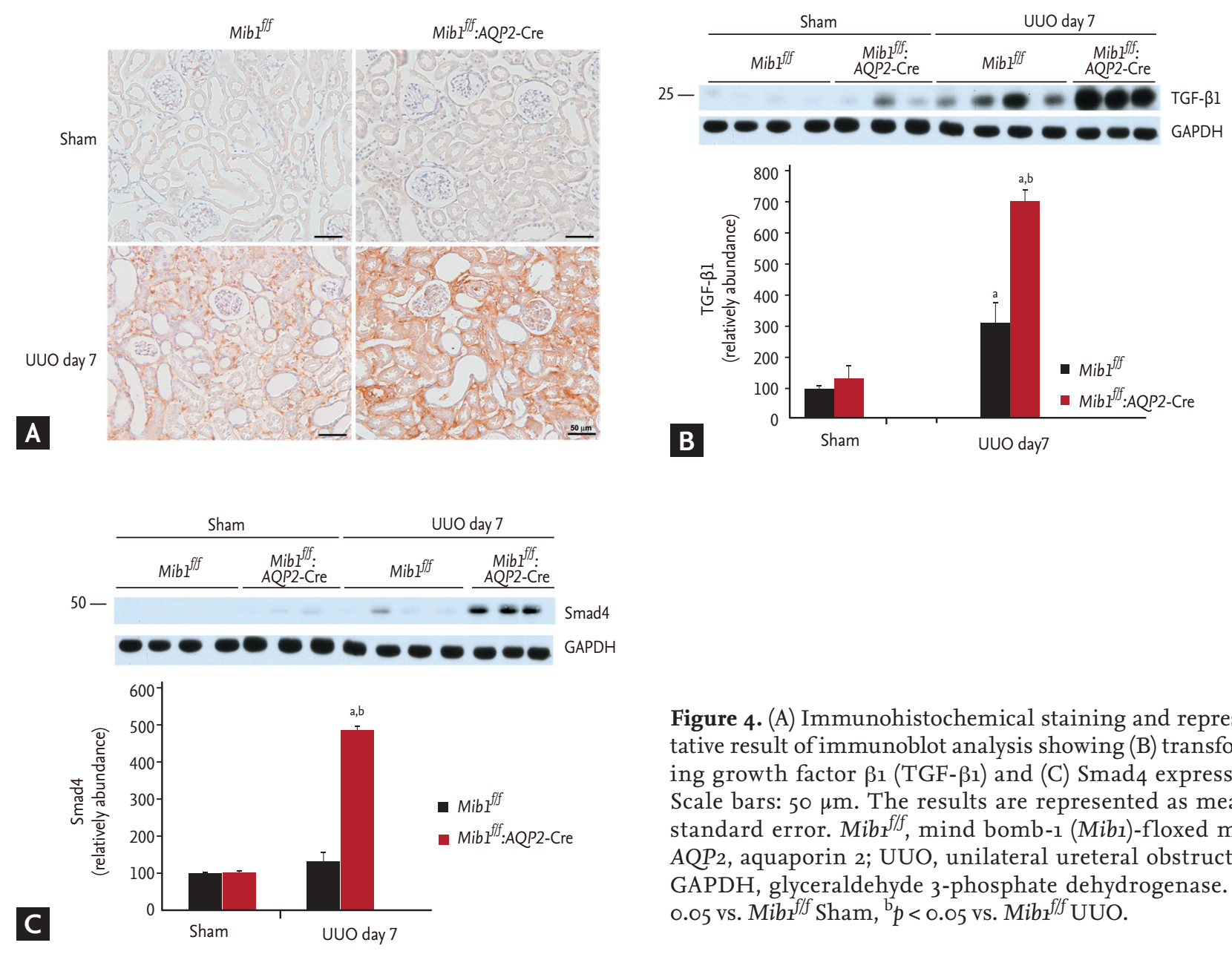

Figure 4. (A) Immunohistochemical staining and representative result of immunoblot analysis showing (B) transforming growth factor $\beta 1$ (TGF- $\beta 1$ ) and (C) Smad 4 expression. Scale bars: $50 \mu \mathrm{m}$. The results are represented as mean \pm standard error. Mibs $f / f$, mind bomb-1 (Mibı)-floxed mice; $A Q P 2$, aquaporin 2; UUO, unilateral ureteral obstruction; GAPDH, glyceraldehyde 3-phosphate dehydrogenase. ${ }^{a} p<$ 0.05 vs. MibI ${ }^{f / f}$ Sham, ${ }^{b} p<0.05$ vs. MibI f/f UUO.

in those of Mibpf/s mice (Fig. ${ }_{5} \mathrm{C}$ ).

\section{DISCUSSION}

Here, we investigated the role of Notch signaling in the collecting duct in renal tubulointerstitial fibrosis. The obtained results demonstrate that Notch signaling in principal cells of the collecting duct plays a role in the development of renal tubulointerstitial fibrosis. The genetic deficiency of Mibr in principal cells led to the upregulation of TGF- $\beta 1$ and promoted renal tubulointerstitial fibrosis after UUO. Additionally, the obtained results indicate that the principal cell-specific Mibr deletion increases ECM deposition and enhances tubulointerstitial fibrosis after UUO. The results obtained by investigating the expression of Notch 1 in Mibr deficient sham-operated or UUO mice showed that Mibı

is a potential regulator of Notch signaling pathway, and that the induction of Notch 1 is inhibited in mice with principal cell-specific Mibı deletion.

The precise mechanism of Mibl-mediated regulation of renal tubulointerstitial fibrosis is unclear [19]. Several possible mechanisms have been suggested. UUO may result in a distal nephron injury. Furthermore, UUO may alter the structure and cellular composition of the collecting duct cells, which may result in reduction of AQP2-positive principal cell numbers [19]. Jeong et al. [11] reported that Mibr deficiency in ureteric bud (Mibfff:Hoxby-Cre ${ }^{+}$mice) leads to the inactivation of Notch signaling during the development of renal collecting duct, which inhibits principal cell differentiation from precursor cells and diminishes the number of principal cells. Additionally, Notch signaling pathway was reported to be activated after acute ischemic injury, which requires the regeneration of tubular cells in order to re- 

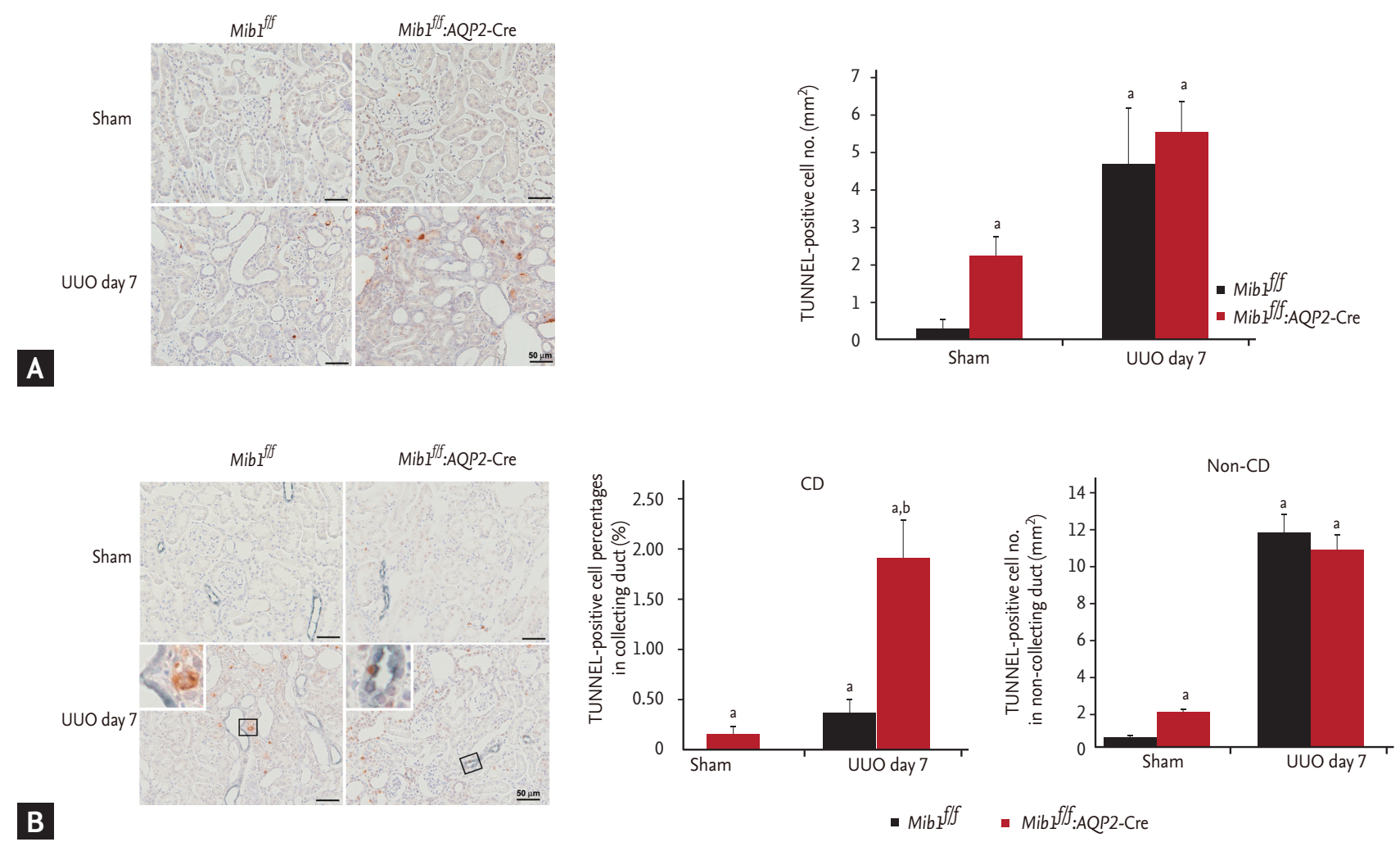

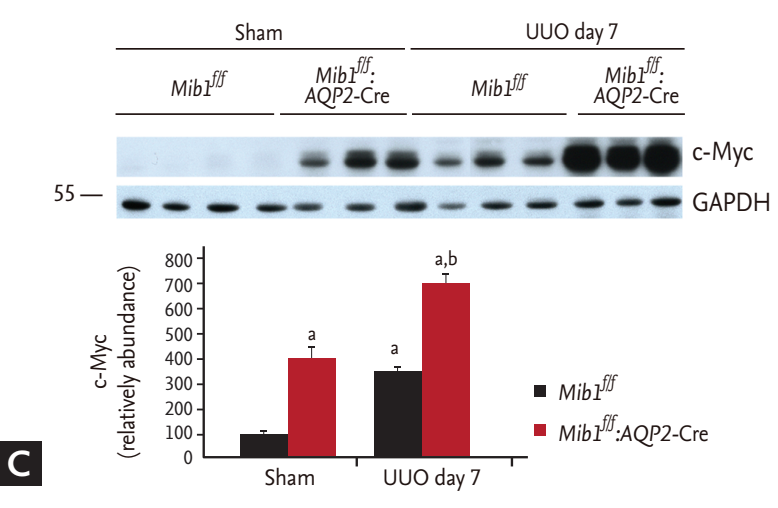

Figure 5. (A) TUNNEL (Terminal deoxynucleotide transferase dUTP Nick End Labeling) assay results and the quantification of TUNNEL-positive cells, (B) aquaporin 2 (AQP2) (blue) and TUNNEL (brown) double-staining is presented, with TUNNEL positive cell percentage in the collecting duct (\%) and TUNNEL-positive cell number in non-collecting duct $\left(/ \mathrm{mm}^{2}\right)$. (C) Analysis of c-Myc expression. Scale bars: $50 \mu \mathrm{m}$. The results are presented as mean \pm standard error. Mibs ${ }^{\mathrm{f} / \mathrm{f}}$, mind bomb-1 (Mibı)-floxed mice; UUO, unilateral ureteral obstruction; CD, collecting duct; GAPDH, glyceraldehyde 3-phosphate dehydrogenase. ${ }^{\mathrm{a}} \mathrm{p}<0.05 \mathrm{vs}$. Mibr ${ }^{\mathrm{f} / \mathrm{f}} \mathrm{Sham}$, ${ }^{\mathrm{b}} \mathrm{p}<0.05 \mathrm{vs}$. MibI ${ }^{\mathrm{f} / \mathrm{f}} \mathrm{UUO}$. store the integrity of the tubular epithelium [20]. Therefore, the inactivation of Notch signaling in principal cells of Mibı-deficient mice may lead to an insufficient repair of principal cells after UUO. Furthermore, as recent studies show, using in vitro models $[15,16]$ and fetal UUO model [14], it was shown that collecting duct cells may be involved in the development of renal tubulointerstitial fibrosis.

Here, we showed that the rate and the number of TUNNEL-positive cells after UUO in the collecting duct were significantly higher, whereas the death rate of noncollecting duct cells was lower in the obstructed kidneys of Miby ff:AQP2-Cre ${ }^{+}$mice than in those of Mibyffs mice (Fig. $5 \mathrm{~A}$ and $5 \mathrm{~B}$ ). The c-Myc overexpression may lead to an increase in apoptosis [21-23]. Our finding that c-Myc expression is significantly higher in the obstructed kidneys of Mibrff: $A \mathrm{QP}_{2}-\mathrm{Cre}^{+}$mice than in those of Mibsfls mice may indicate that the apoptotic effect of Mibr in renal principal cell after UUO may be regulated by c-Myc. The obtained data show that the increase in cell death rate in collecting ducts may be one of the underlying mechanisms of renal tubulointerstitial fibrosis development. Further studies to elucidate the precise mechanism are needed. 
The results of this study are inconsistent with a previous study, which reported that genetic deletion and pharmacologic inhibition of the members of the Notch signaling pathway in proximal tubules reduce renal tubulointerstitial fibrosis [13]. Although the reason for this disagreement between the results of this study and the results we obtained is unclear, we suggest that it may be due to the differences in the experimental design. Cellular response after UUO may differ between proximal and distal nephrons [24]. In response to UUO, superoxide molecules accumulate in proximal tubules, causing apoptosis and necrosis, which is followed by progressive atrophy and collapse of proximal tubules. The rapid proximal tubular damage in response to UUO injury may be a primary determinant of renal parenchymal loss, which may result in progressive increase in ECM deposition. In contrast to the proximal tubules, the distal nephrons may have adaptive responses to the UUO injury. In distal nephrons, collecting ducts dilate and undergo cellular remodeling, in order to preserve distal tubular integrity and maintain their patency [24]. In this study, we used the principal cells-specific Mibı deficient mice, while Bielesz et al. [13] used the proximal tubules-specific Notch 1 deficient mice (Rbpjf/s:PEPCK-Cre ${ }^{+}$ mice). Notch signaling pathway may play different roles in the segment-specific nephron responses to UUO injury, which should be investigated in further studies.

In conclusion, these results suggest that the inactivation of Mib1, an E3 ligase expressed by the ligand-expressing cells, is required for efficient Notch activation, which leads to increased renal tubulointerstitial fibrosis and apoptosis of principal cells after UUO.

\section{KEY MESSAGE}

1. Mind bomb-1 (Mibs) is a potential regulator of Notch signaling pathway.

2. Notch signaling in the collecting duct plays an important role in the regulation of renal tubulointerstitial fibrosis after unilateral ureteral obstruction.

3. The increase in cell death rate in collecting ducts may be one of the underlying mechanisms of renal tubulointerstitial fibrosis development.

\section{Conflict of interest}

No potential conflict of interest relevant to this article was reported.

\section{Acknowledgments}

This research was supported by Basic Science Research Program through the National Research Foundation of Korea (NRF) funded by the Ministry of Education, Science and Technology (NRF-2015R1D1A1A09059195) and MRC for Cancer Evolution Research Center (2012R1A5A2047939), and the institute of Clinical Medicine Research of Bucheon St. Mary's Hospital Research Fund, BCMC14IAo1.

\section{REFERENCES}

1. Kriz W, Elger M, Hosser H, et al. How does podocyte damage result in tubular damage? Kidney Blood Press Res 1999;22:26-36.

2. Sutariya B, Jhonsa D, Saraf MN. TGF- $\beta$ : the connecting link between nephropathy and fibrosis. Immunopharmacol Immunotoxicol 2016;38:39-49.

3. Yan J, Zhang Z, Jia L, Wang Y. Role of bone marrow-derived fibroblasts in renal fibrosis. Front Physiol 2016;7:61.

4. Nath KA. Tubulointerstitial changes as a major determinant in the progression of renal damage. Am J Kidney Dis 1992;20:1-17.

5. Eddy AA. The origin of scar-forming kidney myofibroblasts. Nat Med 2013;19:964-966.

6. Kopan R, Ilagan MX. The canonical Notch signaling pathway: unfolding the activation mechanism. Cell 2009;137:216-233.

7. Le Borgne R. Regulation of Notch signalling by endocytosis and endosomal sorting. Curr Opin Cell Biol 2006;18:213-222.

8. Boulianne GL, de la Concha A, Campos-Ortega JA, Jan LY, Jan YN. The Drosophila neurogenic gene neuralized encodes a novel protein and is expressed in precursors of larval and adult neurons. EMBO J 1993;12:2586.

9. Itoh M, Kim CH, Palardy G, et al. Mind bomb is a ubiquitin ligase that is essential for efficient activation of Notch signaling by delta. Dev Cell 2003;4:67-82.

10. Koo BK, Yoon MJ, Yoon KJ, et al. An obligatory role of mind bomb-1 in notch signaling of mammalian development. PLoS One 2007;2:e1221. 
11. Jeong HW, Jeon US, Koo BK, et al. Inactivation of Notch signaling in the renal collecting duct causes nephrogenic diabetes insipidus in mice. J Clin Invest 2009;119:32903300 .

12. Niranjan T, Bielesz B, Gruenwald A, et al. The Notch pathway in podocytes plays a role in the development of glomerular disease. Nat Med 2008;14:290-298.

13. Bielesz B, Sirin Y, Si H, et al. Epithelial Notch signaling regulates interstitial fibrosis development in the kidneys of mice and humans. J Clin Invest 2010;120:4040-4054.

14. Butt MJ, Tarantal AF, Jimenez DF, Matsell DG. Collecting duct epithelial-mesenchymal transition in fetal urinary tract obstruction. Kidney Int 2007;72:936-944.

15. Ivanova L, Butt MJ, Matsell DG. Mesenchymal transition in kidney collecting duct epithelial cells. Am J Physiol Renal Physiol 2008;294:F1238-F1248.

16. Smith JP, Pozzi A, Dhawan P, Singh AB, Harris RC. Soluble HB-EGF induces epithelial-to-mesenchymal transition in inner medullary collecting duct cells by upregulating Snail-2. Am J Physiol Renal Physiol 2009;296:F957-F965.

17. Fujiu K, Manabe I, Nagai R. Renal collecting duct epithelial cells regulate inflammation in tubulointerstitial damage in mice. J Clin Invest 2011;121:3425-3441.

18. Ding Y, Kim SI, Lee SY, Koo JK, Wang Z, Choi ME. Auto- phagy regulates TGF- $\beta$ expression and suppresses kidney fibrosis induced by unilateral ureteral obstruction. J Am Soc Nephrol 2014;25:2835-2846.

19. Hiatt MJ, Ivanova L, Trnka P, Solomon M, Matsell DG. Urinary tract obstruction in the mouse: the kinetics of distal nephron injury. Lab Invest 2013;93:1012-1023.

20. Kobayashi T, Terada Y, Kuwana H, et al. Expression and function of the delta-1/Notch-2/Hes-1 pathway during experimental acute kidney injury. Kidney Int 2008;73:12401250.

21. Pelengaris S, Khan M, Evan G. c-MYC: more than just a matter of life and death. Nat Rev Cancer 2002;2:764-776.

22. Pelengaris S, Khan M. The many faces of c-MYC. Arch Biochem Biophys 2003;416:129-136.

23. Cianfanelli V, Fuoco C, Lorente M, et al. AMBRAı links autophagy to cell proliferation and tumorigenesis by promoting c-Myc dephosphorylation and degradation. Nat Cell Biol 2015;17:20-30.

24. Forbes MS, Thornhill BA, Minor JJ, Gordon KA, Galarreta CI, Chevalier RL. Fight-or-flight: murine unilateral ureteral obstruction causes extensive proximal tubular degeneration, collecting duct dilatation, and minimal fibrosis. Am J Physiol Renal Physiol 2012;303:F120-F129. 